\title{
Frecuencia de intervenciones quirúrgicas realizadas en el Hospital General de Jaén, Perú, 2018
}

\section{Frequency of surgical interventions carried out at the general Hospital of Jaén, Peru, 2018}

\author{
Graciela Puerta Santillán ${ }^{1}$, Yudelly Torrejón Rodríguez²
}

\section{RESUMEN}

El objetivo de la presente investigación fue determinar la frecuencia de intervenciones quirúrgicas realizadas en el Hospital General de Jaén, Perú, 2018. Para la muestra se tuvo en cuenta el promedio según el historial de las intervenciones quirúrgicas realizadas en los tres últimos años. Se encontró que el 55\% corresponde a cirugías mayores y el 44,6 corresponde a cirugías menores; son más frecuentes las cirugías ginecoobstétricas, con el $28,9 \%$, el $4,3 \%$ corresponde a cirugías pediátricas y el $0.6 \%$ a cirugías urológicas. El $42.9 \%$ corresponde a cirugías de emergencia y el $57.1 \%$ corresponde a cirugías de urgencias. En conclusión existe similitud porcentual en la frecuencia de las intervenciones quirúrgicas tanto en cirugías mayores y menores, realizadas en el Hospital General de Jaén. Las cirugías ginecoobstétricas fueron las más frecuentes realizadas durante el año 2018 y las cirugías de emergencia son las más frecuentes en comparación a las cirugías de urgencia.

Palabras clave: Frecuencia, intervenciones quirúrgicas, hospital

\begin{abstract}
The objective of this research was to determine the frequency of surgical interventions performed at the General Hospital of Jaén, Peru, 2018. For the sample, the average was taken into account according to the history of the surgical interventions carried out in the last three years. It was found that 55\% correspond to major surgeries and 44.6 correspond to minor surgeries; gynecology-obstetric surgeries are more frequent, with $28.9 \%, 4.3 \%$ correspond to pediatric surgeries and $0.6 \%$ to urological surgeries. $42.9 \%$ correspond to emergency surgeries and $57.1 \%$ correspond to emergency surgeries. In conclusion, there is a percentage similarity in the frequency of surgical interventions both in major and minor surgeries, performed at the General Hospital of Jaén. Gynecological and obstetric surgeries were the most frequent performed during 2018 and emergency surgeries are the most frequent compared to emergency surgeries.
\end{abstract}

Keywords: Frequency, surgical interventions, hospital

\footnotetext{
${ }^{1}$ Bachiller de la Escuela Profesional de Enfermería, de la Facultad de Ciencias de la Salud de la Universidad Nacional Toribio Rodríguez de Mendoza de Amazonas. Correo electrónico:grachiix_96@hotmail.com

${ }^{2}$ Magister. Docente del Departamento Académico de Salud Pública de la Facultad de Ciencias de la Salud. Correo electrónico: yudelly.torrejón@untrm.edu.pe
} 


\section{INTRODUCCIÓN}

Las intervenciones quirúrgicas en el mundo son de gran relevancia, sobre todo si nos referimos a las cirugías mayores, vale decir apendicetomías, colecistectomías, cesáreas, hernias. Las intervenciones quirúrgicas ya sea referente a cirugía mayores y menores tiene relación con la escalada de violencia, tal es así por ejemplo que en el noroeste de África ha acaparado la atención mundial en los últimos tiempos, las noticias diarias de lesiones y muertes procedentes de la región suscitan preocupación. Sin embargo, lo que no siempre trasciende es el hecho de que muchos de esos países carecen de la capacidad quirúrgica necesaria para curar a sus heridos, y esta incapacidad de proporcionar cuidados quirúrgicos está contribuyendo a un aumento sustancial del número de víctimas mortales. El $90 \%$ de las muertes por traumatismo acaecen en países de ingresos bajos y medios (Montejo, 2015, p. 299).

Este dato no es sorprendente si tenemos en cuenta que al tercio más pobre de la población mundial tan sólo le corresponde el 3,5\% de las operaciones quirúrgicas practicadas en todo el mundo. Muchos hospitales de esos países no tienen abastecimiento seguro de agua potable, oxígeno, electricidad o anestesias, lo que hace sumamente difícil que se puedan practicar en ellos hasta las operaciones quirúrgicas más básicas. (Alfonso R, 2016, p. 375).

Pese a este desequilibrio mundial en materia de capacidad quirúrgica, la cirugía sigue siendo "el hijastro olvidado del sector de la salud mundial". Ninguna organización mundial de financiación se centra específicamente en la prestación de cuidados quirúrgicos, y ninguno de los principales donantes están dispuestos a apoyar y reconocer la cirugía como un aspecto imprescindible de la salud pública mundial. Esto obedece principalmente a las siguientes ideas erróneas sobre la cirugía que no están basadas en la verdad. (Mederos, 2016, p. 34).

En primer lugar, mucha gente cree que la atención quirúrgica sólo es aplicable a una parte muy limitada de la carga mundial de morbilidad y que por ello no es una prioridad. En realidad, cada año, los traumatismos son responsables de la muerte de más de cinco millones de personas en todo el mundo, es decir, de casi una de cada diez muertes. Muchas de las víctimas son el principal sostén de la familia. Según un estudio reciente, un tercio de las defunciones por traumatismo afectan al grupo de edad de 15-44 años, que es el segmento económicamente más productivo de la población. Además, la función de la atención quirúrgica va más allá del tratamiento de los traumatismos. La cirugía es uno de los elementos clave de la atención primaria e incluye la gestión de luxaciones traumáticas, el tratamiento de fracturas abiertas para prevenir la osteomielitis y el drenaje de abscesos.
(Maristany, 2010, p. 47).

También es una intervención esencial para limitar la mortalidad materna e infantil. En un informe reciente de la OMS se calcula que, en todo el mundo, alrededor de 260000 defunciones se debieron a anomalías congénitas y 342900 a la mortalidad materna. Una parte importante de estas muertes podrían haberse evitado aplicando cuidados quirúrgicos simples y eficaces en función del costo. En segundo lugar, se suele creer que la atención quirúrgica es demasiado onerosa para ser brindada como intervención de salud pública. Sin embargo, la cirugía puede ser sumamente eficaz en relación con el costo, incluso si se compara con intervenciones no quirúrgicas que suelen aplicarse como medidas de salud pública. (Viamontes, 2015, p. 111).

Como señaló recientemente Paul Farmer, cofundador de la organización internacional Partners in Health, "la salud mundial no tiene por qué ser una carrera competitiva para procurarse recursos escasos... podemos construir un movimiento coherente que incluya en su seno a la cirugía". La OMS debería ejercer su liderazgo y contribuir a que la atención quirúrgica adquiera un papel más relevante en el ámbito de la salud mundial, y debería organizar planes de acción para abordar las necesidades de capacidad quirúrgica hasta ahora desatendidas.

En Perú, existe el $89 \%$ de los pacientes que son intervenidos quirúrgicamente son corresponden a cirugías mayores de las cuales el $57 \%$ de las mismas son realizadas con laparoscopía, lo que implica en las personas un gran beneficio de debido a que estas cirugías al no existir complicaciones ayudan a que los pacientes se recuperen de manera rápida reinsertándose a sus labores administrativas después del tercer día de haber sido intervenidos quirúrgicamente.

El presente trabajo de investigación se realizó porque las intervenciones quirúrgicas se presenta definitivamente en todas las ciudades donde se cuenta con hospitales con capacidad resolutiva para realizar las cirugías ya sea mayores y menores y según su complejidad y especialidad éstas se derivan a un nosocomio de mayor competencia según el caso, por lo que se torna de vital importancia conocer la frecuencia respecto a las intervenciones quirúrgicas realizadas en el Hospital General de Jaén (H.G.J.) a fin de conocer cuáles son las más frecuentes como diagnóstico real y/o como base de datos.

En tal sentido con los resultados obtenidos en esta investigación permitirá conocer la frecuencia de las intervenciones quirúrgicas; así mismo servirá en la toma de decisiones respecto a la gestión de nuevos profesionales de enfermería con la especialidad en centro quirúrgico y como base de datos para posteriores investigaciones. 
Considerando el quehacer del profesional de enfermería está basado en el área docente, asistencial, administrativa e investigación; por lo tanto, cabe resaltar la importancia en el desempeño de sus funciones en el centro quirúrgico.

Por las razones antes mencionadas es que nos proponemos determinar la frecuencia de intervenciones quirúrgicas realizadas en el Hospital General de Jaén, 2018, identificar la frecuencia de intervenciones quirúrgicas según especialidades: ginecoobstétricia, general, pediátrica y urológica realizadas e identificar la frecuencia de intervenciones quirúrgicas según dimensiones: emergencias y urgencias realizadas en el Hospital General de Jaén-2018.

\section{MATERIAL Y MÉTODO}

Método: La investigación fue de enfoque cuantitativo, de nivel descriptivo; de tipo: Según la intervención de la investigadora fue observacional, según la planificación de la toma de datos fue retrospectivo. (Supo, 2014, p,55).

\section{Técnica e instrumentos de recolección de datos.}

Se utilizó el instrumento de registro o lista de cotejo para la recolección de datos.

\section{RESULTADOS}

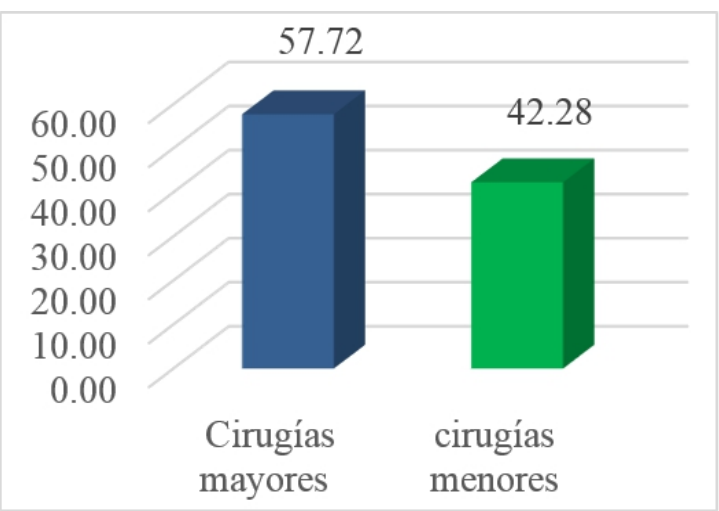

Figura 1. Porcentaje de intervenciones quirúrgicas realizadas en el Hospital General de Jaén, 2018.

Descripción: El $57.72 \%$ corresponde a cirugías mayores y el $42,28 \%$ a cirugías menores.

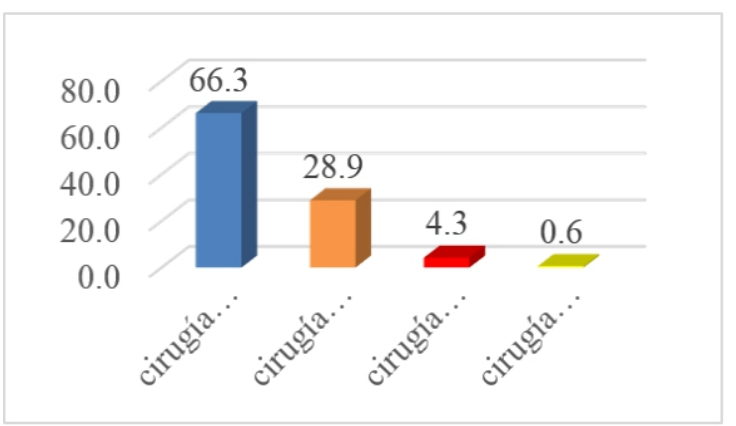

Figura 2. Porcentje de intervenciones quirúrgicas según especialidades: gineco obstétrica, general, pediátrica y urológica realizadas en el Hospital General de Jaén.

Descripción: El $66.3 \%$ corresponde a cirugías gineco obstétricas, el 28,9\% a cirugías generales, el 4,3\% corresponde a cirugías pediátricas y el $0.6 \%$ a cirugías urológicas.

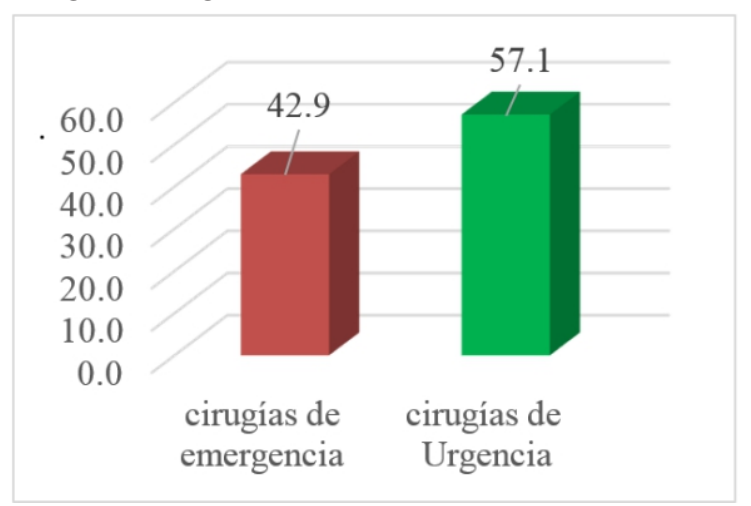

Figura 3. Porcentaje de intervenciones quirúrgicas según dimensiones: emergencia y urgencias realizadas en el Hospital General de Jaén, 2018.

Descripción: El $42.9 \%$ corresponde a cirugías de emergencia y el $57.1 \%$ corresponde a cirugías de urgencias.

\section{DISCUSIÓN}

En la figura 1, se muestra que las cirugías que se realizaron fueron que del 100\% (4253) del total de cirugías realizadas, el 57.72\% (2455) corresponde a cirugías mayores y el 42,28\% (1798) a cirugías menores.

Cuya, J. (2015), México, realizó la investigación frecuencia de las intervenciones quirúrgicas convencionales y no convencionales en el Hospital General de México (HGM), obteniéndose como resultado que el $61 \%$ corresponden a cirugías convencionales, destacándose las cirugías de ginecología y el 39\% corresponden a las cirugías no convencionales, sobresaliendo con mayor frecuencia las COLELAP. Las cirugías convencionales se realizan con mayor frecuencia en el HGM, al respecto, se deduce que no existe similitud, en primer lugar, en el Hospital General de Jaén, no se realizaban en el 2018 cirugías no convencionales o laparoscópicas, se deducía que realmente es necesario realizar este tipo de intervenciones quirúrgicas por tener éstas ventajas muy marcadas para la persona que necesariamente tiene que ser intervenida, este tipo de cirugías, no afecta mucho la integridad de los tejidos de los pacientes por caracterizarse con la realización de pequeñas incisiones a nivel de la piel, por donde se insertan los trocares, la cámara, el instrumental quirúrgico 
(pinzas hemostáticas, de corte y disección, de diéresis, etc.), por lo que la recuperación del paciente es más acelerada y el alta de la persona no pasa del tercer día de haber sido intervenido, excepcionalmente sí que no presentase por supuesto complicaciones.

Callozos, C. (2015) Colombia. Realizó la investigación, frecuencia de las intervenciones quirúrgicas convencionales y no convencionales en el Hospital General de Medellín (HGM), incluyeron todos los pacientes de cirugía mayor del HGM atendidos en febrero y marzo de 2015, obteniéndose como resultado que el $58 \%$ corresponden a cirugías convencionales, destacándose las cirugías de apéndice y el $42 \%$ corresponden a las cirugías no convencionales, sobresaliendo con mayor frecuencia las COLELAP. Las cirugías convencionales se realizan con mayor frecuencia en el HGM.

Comparando con nuestros resultados obtenidos, se evidencia de igual forma que no existe similitud, por las cirugías no convencionales que se realizan en el Hospital General de Medellín, sin embargo, la implementación de equipos de laparoscopía para la realización de algunas cirugías mayores, es de vital importancia, hoy en día, en la mayoría de ciudades de los diferentes países del mundo realizan las cirugía no convencionales o laparoscópicas, el desarrollo de la ciencia en el campo de la salud para la rehabilitación y recuperación de las personas va de la mano con los avances científicos que día a día se vienen realizando, siendo sus aportes sorprendentes, evidentemente estamos atravesando y viviendo en la era de la globalización y del avance tecnológico que contribuye en este caso al desarrollo de la ciencia en el área de la salud.

En la figura 2, se obtuvo que del 100\% (4253), 66.3\% (2819) corresponde a cirugías gineco obstétricas, el $28,9 \%$ (1227) a cirugías generales, el 4,3\% (181) corresponde a cirugías pediátricas y el $0.6 \%$ (26) a cirugías urológicas.

Al respecto, Pérez, J. (2015), en Cuba realizó una investigación sobre frecuencia de las intervenciones quirúrgicas mayores, que tuvo como objetivo describir los resultados obtenidos con la colecistectomía convencional abierta, en el tratamiento de la litiasis vesicular, se encontró, operados 147 pacientes; predominó el grupo de 41 a 60 años y el sexo femenino; el 65\% correspondió a la especialidad de cirugía general, el $10 \%$ a cirugía pediátrica, el $9 \%$ a cirugías urológicas, y el $16 \%$ correspondieron a cirugías ginecoobstétricas; el dolor en hipocondrio derecho y la intolerancia a los alimentos grasos y granos, fueron los síntomas y signos que prevalecieron. La cefazolina fue el antibiótico más utilizado. Los pacientes evolucionaron de forma satisfactoria, las complicaciones fueron mínimas. La colecistectomía convencional abierta sigue siendo una opción favorable para el tratamiento de la litiasis vesicular, ante la ausencia de tecnología de avanzada.

Al realizar la contrastación con los resultados del estudio obtenidos, se muestra que no existe similitud al respecto, se ha evidenciado que son las cirugías ginecoobstétricas las que predominan con mayor frecuencia con un $29 \%$, se tendría que investigar las razones por la cual las cirugías ginecoobstétrica no es más notorio su porcentaje en el caso del estudio de Álvarez, talvez se relacione con la falta o deficiente recurso humano referente a los médicos especialistas en ginecología, por otro lado es necesario investigar otros factores que tengan que ver con la accesibilidad, la presencia de otros establecimientos de salud cercanos con capacidad resolutiva para realizar éste tipo de cirugías.

Alvarado, J. (2016) Chile, realizó su investigación registro de las intervenciones quirúrgicas mayores; tuvo como objetivo, comunicar un análisis descriptivo de las intervenciones quirúrgicas realizadas por los residentes del Programa de Cirugía General (PCG) de la Pontificia Universidad Católica de Chile (PUC) en rol de primer cirujano. El promedio de intervenciones realizadas por residente fue de 481 ( $20 \%$ en 1 er año). Las más frecuentes fueron: colecistectomía laparoscópica $(115 ; 24 \%)$, apendicectomía clásica $(89 ; 19 \%)$, hernioplastía (43;9\%), apendicectomía laparoscópica $(34 ; 7 \%)$ y colecistectomía clásica $(25 ; 5 \%)$. Las operaciones de especialidades derivadas que más se realizaron: colectomía $(12 ; 2 \%)$; tiroidectomíaparatiroidectomía $(9 ; 2 \%)$, accesos vasculares $(8 ; 2 \%)$, toracotomía-VATSesternotomía $(5 ; 1 \%)$ y cirugía mamaria $(4 ; 1 \%)$. Un $53 \%$ de las intervenciones fueron de urgencia, observándose una inversión de la proporción entre procedimientos electivos vs de urgencia a lo largo de la formación (62 vs $38 \%$ en 1 ro y 34 vs $66 \%$ en 3ro; p < 0,002), de las intervenciones abdominales (366), 58\% fue por laparotomía y $42 \%$ por laparoscopia, un $74 \%$ y $26 \%$ de todas las intervenciones se realizaron en centros de

Santiago y regiones, respectivamente. Conclusiones: Los residentes de la PUC completan un volumen importante de intervenciones en rol de primer cirujano durante los 3 años de su PCG, superando las exigencias mínimas establecidas.

Al comparar con los resultados de la presente investigación, se evidencia de igual modo que no hay similitud, se deduce que, en el estudio de Alvarado, realizado en la Pontificia Universidad Católica de Chile (PUC), es un nosocomio, de mayor complejidad y capacidad resolutiva con mayor especialidad en comparación con las cirugías realizadas en el Hospital General de Jaén, las apendicetomías fueron las más frecuentes, siendo en el presente estudio las cirugías gineco obstétricas. En el Hospital General de Jaén predominan frecuentemente este tipo de cirugías por ser un 
Hospital referencial ubicado estratégicamente en esta zona nororiental del país, que acceden pacientes referidos de toda la Provincia de San Ignacio, de toda la Provincia de Jaén, consecuentemente influye de igual modo la cantidad de gestantes y el alto índice de natalidad.

En la figura 3, se obtuvo que del $100 \%$ (4253) de cirugías realizadas, el 42.9\% (1826) corresponde a cirugías de emergencia y el $57.1 \%$ (2427) corresponde a cirugías de urgencias.

Alvarado, J. (2016) Chile, realizó su investigación registro de las intervenciones quirúrgicas mayores; tuvo como objetivo: comunicar un análisis descriptivo de las intervenciones quirúrgicas realizadas por los residentes del Programa de Cirugía General (PCG) de la Pontificia Universidad Católica de Chile (PUC) en rol de primer cirujano. Un 53\% de las intervenciones fueron de urgencia, observándose una inversión de la proporción entre procedimientos electivos vs de urgencia a lo largo de la formación (62 vs $38 \%$ en 1 ro y 34 vs $66 \%$ en 3 ro; $p<0,002)$, de las intervenciones abdominales (366), 58\% fue por laparotomía y $42 \%$ por laparoscopia, un $74 \%$ y $26 \%$ de todas las intervenciones se realizaron en centros de Santiago y regiones, respectivamente. Conclusiones: Los residentes de la PUC completan un volumen importante de intervenciones en rol de primer cirujano durante los 3 años de su PCG, superando las exigencias mínimas establecidas.

Al respecto, se deduce que sí existe similitud en el resultado de ambas investigaciones, en ambos hospitales se realizaron intervenciones quirúrgicas mayores y menores con porcentajes con poco margen de diferencia, realmente se ha experimentado siempre que la frecuencia de las cirugías menores también son abundantes, y que además influye el corto tiempo de realización del procedimiento que se emplea para realizarlas, mayormente son procedimientos invasivos menores con corto tiempo en comparación de las cirugías menores, que pueden realizarse en el tópico de emergencia, en los consultorios externos de cirugía preferentemente, como extracciones de uñas, de cuerpos extraños, lipomas, debridaciones, etc.

\section{CONCLUSIONES}

1. Existe similitud porcentual en la frecuencia de las intervenciones quirúrgicas tanto en cirugías mayores y menores, realizadas en el Hospital General de Jaén.

2. Las cirugías ginecoobstétricas fueron las más frecuentes realizadas durante el año 2018.

3. Las cirugías de emergencia son más frecuentes en comparación a las cirugías de urgencias.

\section{REFERENCIAS BIBLIOGRÁFICAS}

Alfonso RF. Mortalidad geriátrica en cirugía general. Rev Cubana Cir 2016; 26(3):375-85.

Alvarado, J. (2016). Intervenciones realizadas por recidentes cirugia $g$ e $n$ e $r$ a $l$. http://repositorio.uta.edu.ec/handle/123456 $789 / 6596$.

Callazos, C. (2012). Verificación de la lista de chequeo para seguridad en cirugia desde la perspectiva del paciente.colombia. http://www.revcolanest.com.co/es/verificaci o n - 1 i s t a - c h e q u e o - s e g u r i d a d cirugia/articulo/90199316/.

Hines JR, Harris CD. Colostomía y cierre de colostomías. Clin $\mathrm{Q} \quad \mathrm{u} \quad \mathrm{i} \quad \mathrm{r}$ Norteam 2017;(dic):1379.

Maristany GC. Cirugía mayor en el anciano. Rev Cubana Cir 2015;23(5):445-58.

Montejo VN, Hernández GA, Montalvo SL, Vilorio HPL. Cáncer de colon, estudio comparativo de anastomosis en 1 y 2 planos, en 175 pacientes. Rev Cubana Cir 2015;29(2):299308.

Mederos CON, Viamontes F, Carvhalo F, Alburqueque M. Sutura contínua extramucosa en lesiones por heridas de armas de fuego. Rev Cubana Cir 2016;34(2):107-10.

Newton M. Complication of gynecologic surgery Artz-Hardy. Complication in surgery and their management. La Habana: Instituto Cubano del Libro, 2017:665.

Pérez, J. L. (2015). Colecistectomía convencional abierta en el tratamiento quirurgico de la

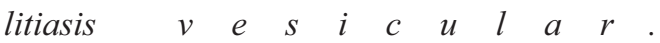
http://scielo.sld.cu/scielo.php?script=sci_art text\&pid=S1727-897X2015000100004.

Rodríguez FZ. Mortalidad en el paciente de edad avanzada en cirugía general. Rev Cubana Cir 2018;27(4):16-26.

Torres BA. Drenaje en apendicitis perforada. Rev Cir Esp 2016;175(1):33-4.

Vallejo Witowska R. Abdomen agudo en paciente geriátrico. Rev Cubana Cir 2015;22(4):326-37.

Viamontes F, Mederos CON, Carvhalo F, Trujillo ME. Uso sistemático de la sutura continua en la cirugía de colon. Rev Cubana Cir 2015;34(2):111-4. 